\title{
Generation of highly differentiated BHY oral squamous cell carcinoma multicellular spheroids
}

\author{
DENISE SIEVERS ${ }^{1}$, JENS BUNZENDAHL ${ }^{1}$, ALICE FROSCH ${ }^{2}$, CHRISTINA PERSKE $^{2}$, \\ BERNHARD HEMMERLEIN $^{2,3}$, HENNING SCHLIEPHAKE ${ }^{1}$ and PHILLIPP BROCKMEYER ${ }^{1}$ \\ Departments of ${ }^{1}$ Oral and Maxillofacial Surgery, and ${ }^{2}$ Pathology, University Medical Centre Goettingen, \\ D-37075 Goettingen; ${ }^{3}$ Institute of Pathology, Helios Klinikum Krefeld, D-47805 Krefeld, Germany
}

Received September 18, 2017; Accepted November 10, 2017

DOI: $10.3892 / \mathrm{mco} .2017 .1514$

\begin{abstract}
Three-dimensional (3D) multicellular spheroids (MCS) are considered suitable models in cancer research and anticancer drug development. Although studying the complex tumour characteristics from all different degrees of malignancy is vital, MCS generation has only been described in a few moderately- and poorly differentiated oral squamous cell carcinoma (OSCC) cell lines. No previous study has demonstrated the MCS formation in a highly differentiated OSCC cell line. For the first time, the present study aimed to generate MCS from the highly differentiated OSCC cell line BHY. BHY spheroids were grown in three independent experiments in 96-well plates through the use of the liquid overlay technique. Although BHY cells are grow slowly and are difficult to culture, they formed compact MCS within $24 \mathrm{~h}$. After 3 days of incubation, no further increase in spheroid size was observed. MCS were harvested, paraffin-embedded and $2 \mu \mathrm{m}$ tissue sections were prepared for further analysis. The diameter and volume of each spheroid were determined. BHY MCS diameter ranged between 46.76 and $233.26 \mu \mathrm{m}$, with a volume range from $5.35 \times 10^{4}-6.65 \times 10^{6} \mu \mathrm{m}^{3}$. In conclusion, using the liquid overlay technique, the highly differentiated OSCC cell line BHY forms different sized spheroids, which may be used for further investigations.
\end{abstract}

\section{Introduction}

Three-dimensional (3D) cell culture models are considered an important tool in cancer research (1-3) and anticancer drug development (2). Owing to intercellular communication and cell-matrix interactions (3-5), multicellular spheroids (MCS) reproduce the in vivo situation more effectively than two-dimensional (2D) monolayer cell culture systems.

Correspondence to: Dr Phillipp Brockmeyer, Department of Oral and Maxillofacial Surgery, University Medical Centre Goettingen, 40 Robert-Koch Street, D-37075 Goettingen, Germany

E-mail: ph.brockmeyer@gmail.com

Key words: multicellular spheroid, MCS, 3D cell culture, oral squamous cell carcinoma, OSCC, BHY
MCS can be used to evaluate cell proliferation, apoptosis, differentiation, gene expression and metabolism, and the tumour response to radiochemotherapy (4). Moreover, MCS can imitate the biological properties of tumour invasion and micrometastasis $(4,6)$. There are several known techniques for spheroid generation, e.g. hanging drop $(7,8)$. The liquid overlay technique has been described as the most suitable technique for reproducible spheroid preparation (3). On a non-adhesive surface, cells adhere to each other and start forming MCS $(5,9,10)$. The generation of MCS has been described in few moderately- and poorly-differentiated oral squamous cell carcinoma (OSCC) cell lines (11). Although studying the complex tumour characteristics from all the different degrees of malignancy is vital, no previous study demonstrating the MCS formation in a highly differentiated OSCC cell line has been conducted. In the present investigation, using the liquid overlay technique, we generate MCS from highly differentiated OSCC cell line BHY for the first time.

\section{Materials and methods}

Cell culture. Highly differentiated OSCC cell line BHY was obtained from the German Collection of Cell Cultures and Microorganisms (DSZM, Braunschweig, Germany). Cell line was cultivated in 90\% Dulbecco's MEM (4.5 g/l glucose) with $10 \%$ h.i. FBS and $1 \%$ Penicillin/Streptomycin in a humidified incubator at $37^{\circ} \mathrm{C}$ with $5 \% \mathrm{CO}_{2}$.

Tumour doubling time. For calculation of the tumour doubling time, 1,1 million cells were seeded in a $25 \mathrm{~cm}^{2}$ cell culture flask and cell density was counted after 48 and $72 \mathrm{~h}$, respectively.

Spheroid preparation. A total of $50 \mu \mathrm{l}$ of the agarose solution ( $0.15 \mathrm{~g}$ of agarose mixed with $10 \mathrm{ml}$ of DMEM) were added to each well of a 96-well microtiter plate, and was let to cool down for $30 \mathrm{~min}$. BHY cell suspension was prepared using a trypsin/EDTA solution. Cells were counted in a Neubauer counting chamber.

In independent experiments 5,000, 10,000 and 15,000 cells per well were used. Since the use of 10,000 and 15,000 cells did not result in the formation of single compact spheroids, only the spheroids with 5,000 initial cells were used for the further studies. 
A total of 480,000 cells in $19.2 \mathrm{ml}$ DMEM were used for spheroid initiation. $200 \mu 1$ of cell suspension was transferred to each well of the agarose-coated 96-well microtiter plate (5,000 cells per well). Since no further increase in sizes could be observed, BHY MCS were harvested after 3 days of incubation, using a pasteur pipette. MCS and its supernatant were transferred from each well into a 50-ml falcon tube, where MCS sedimented. DMEM supernatant was removed, and $20 \mathrm{ml}$ of formalin was added for $5 \mathrm{~h}$ of fixation. Sediment was transferred into a $0.5 \mathrm{ml}$ e-cup, and embedded with $3 \%$ agarose solution. The resulting agarose block was used for paraffin embedding, using the automated embedding workstation Excelsior ES (Thermo Fisher Scientific, Waltham, Massachusetts, USA). All measurements were performed in three independent experiments. Over all, a total of $32 \mathrm{BHY}$ spheroids were used for further evaluation. Two independent investigators evaluated all tissue sections by using a Zeiss Axioskop 2 plus light microscope (Zeiss, Goettingen, Germany).

Evaluation of spheroid size. MCS size was analysed after 3 days on $2 \mu \mathrm{m}$ tissue sections. The diameter of each spheroid was measured by using the AxioVision software (version 4.8; Zeiss, Goettingen, Germany). By assuming a spherical shape, the volume of BHY MCS was calculated using the following equation: $\mathrm{V}=4 / 3 \times \pi \times(\mathrm{d} / 2)^{3}$.

\section{Results}

The analysis of the tumour doubling time of monolayer cell culture revealed a doubling time of $\sim 72 \mathrm{~h}$.

Highly differentiated OSCC cell line BHY formed different sized compact MCS within $24 \mathrm{~h}$ (Fig. 1). After $48 \mathrm{~h}$, spheroids showed a compact body. After $72 \mathrm{~h}$, no further increase in size could be observed. Spheroid compactness decreased with increasing incubation time over three days.

Diameter of the analysed spheroids ranged from $46,76 \mu \mathrm{m}$ to $233,26 \mu \mathrm{m}$ [mean $123.99 \mu \mathrm{m} \pm 59.44$ standard deviation (SD)]. Volume ranged from $5,35 \times 10^{4} \mu \mathrm{m}^{3}$ to $6,65 \times 10^{6} \mu \mathrm{m}^{3}$ [mean $1.74 \times 10^{6} \mu \mathrm{m}^{3} \pm 2.20 \mathrm{SD}$ ] (Table I).

\section{Discussion}

In the present investigation, for the first time, we generated reproducible MCS from highly differentiated OSCC cell line BHY by using the liquid overlay technique.

The advantage of the used method lies in its easy practicability compared to other protocols described in the literature that use the Matrigel, Hanging Drops, or doing Ultra-Low Attachment Assays $(2,11,12)$.

Lee and colleagues compared the spheroid formation of four oral cancer cell lines (SCC9, SCC9 36 , SCC9 36 KDFyn, and SCC9 $36 \mathrm{D} 1$ ) using agarose-coated tissue, and let a $0.6 \%$ agarose solution dry overnight at room temperature (11). The SCC9 36 D1 cell line formed diminutive clusters containing $2-4$ cells. In contrast, the SCC9 36 KDFyn cell line generated large oversized spheres. The aforementioned method to generate spheroids seems to be very sensitive. In our study, the agarose solution was left to cool down at room temperature for only $30 \mathrm{~min}$. An over-dried agarose solution might have a significant influence on spheroid stability.
Table I. Size of each spheroids.

\begin{tabular}{|c|c|c|}
\hline Spheroid (N) & Diameter $[\mu \mathrm{m}]$ & Volume $\left(\mu \mathrm{m}^{3}\right)$ \\
\hline 1 & 115,96 & $8,16 \times 10^{5}$ \\
\hline 2 & 66,76 & $1,56 \times 10^{5}$ \\
\hline 3 & 122,53 & $9,63 \times 10^{5}$ \\
\hline 4 & 233,26 & $6,65 \times 10^{6}$ \\
\hline 5 & 112,72 & $7,50 \times 10^{5}$ \\
\hline 6 & 101,81 & $5,53 \times 10^{5}$ \\
\hline 7 & 208,53 & $4,75 \times 10^{6}$ \\
\hline 8 & 72,74 & $2,02 \times 10^{5}$ \\
\hline 9 & 46,76 & $5,35 \times 10^{4}$ \\
\hline 10 & 97,69 & $4,88 \times 10^{5}$ \\
\hline 11 & 117,79 & $8,56 \times 10^{5}$ \\
\hline 12 & 69,10 & $1,73 \times 10^{5}$ \\
\hline 13 & 129,48 & $1,14 \times 10^{6}$ \\
\hline 14 & 78,87 & $2,57 \times 10^{5}$ \\
\hline 15 & 128,61 & $1,11 \times 10^{6}$ \\
\hline 16 & 227,22 & $6,14 \times 10^{6}$ \\
\hline 17 & 105,55 & $6,16 \times 10^{5}$ \\
\hline 18 & 96,42 & $4,69 \times 10^{5}$ \\
\hline 19 & 217,79 & $5,41 \times 10^{6}$ \\
\hline 20 & 73,10 & $2,05 \times 10^{5}$ \\
\hline 21 & 94,40 & $4,40 \times 10^{5}$ \\
\hline 22 & 55,79 & $9,09 \times 10^{4}$ \\
\hline 23 & 134,75 & $1,28 \times 10^{6}$ \\
\hline 24 & 57,11 & $9,75 \times 10^{4}$ \\
\hline 25 & 66,28 & $1,52 \times 10^{5}$ \\
\hline 26 & 73,17 & $2,05 \times 10^{5}$ \\
\hline 27 & 210,22 & $4,86 \times 10^{6}$ \\
\hline 28 & 211,22 & $4,93 \times 10^{6}$ \\
\hline 29 & 95,29 & $4,53 \times 10^{5}$ \\
\hline 30 & 221,85 & $5,72 \times 10^{6}$ \\
\hline 31 & 209,72 & $4,83 \times 10^{6}$ \\
\hline 32 & 115,26 & $8,02 \times 10^{5}$ \\
\hline
\end{tabular}

A

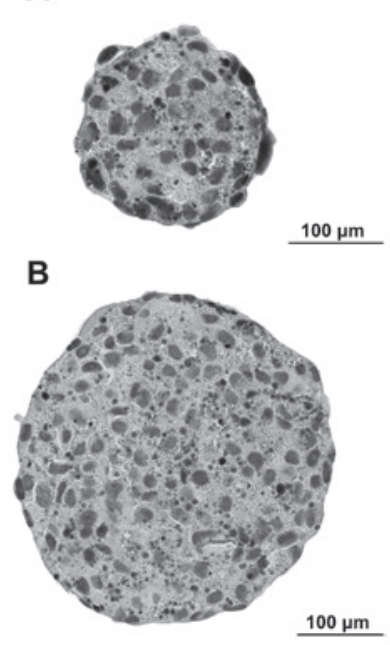

Figure 1. Illustration of different sized BHY spheroids after three days of incubation. (A) Small-sized BHY MCS; (B) Large-sized BHY MCS. MCs, multicellular spheroids. 
The present results are contrary to those described by Adcock and colleagues, who described an increased average spheroid diameter with an increasing initial cell number per well plate, using the CAL-27 cell line from the tongue squamous cell carcinoma (2). While 5,000 BHY cells, resulted in one compact spheroid, adding 10,000 or 15,000 cells to each well plate led to the formation of small aggregates of cells, instead of one compact spheroid in our investigation.

This study shows that it is possible to rapidly generate spheroids with homogeneous size distribution, which is a prerequisite for drug screening $(1,10)$. Within 3 days, BHY MCS grew to $46,76-233,26 \mu \mathrm{m}$ in diameter. This is comparable to the results presented by Adcock et al, where CAL-27 MCS grew to 50-100 $\mu \mathrm{m}$ in diameter within 3 to 4 days (2). Hagemann et al also showed similar results by generating CAL-27 MCS that reached a diameter of 1-3 mm within 5 days (12).

The harvesting procedure of MCS was a critical step in the present protocol. Contrary to the method used by Gebhard et al, who harvested MCS using a plastic pipette of $2 \mathrm{~mm}$ tip diameter and centrifuged them to cell pellets (13), in the present investigation MCS were carefully harvested using a pasteur pipette and were not centrifuged. The use of a plastic pipette and centrifugation disrupted BHY MCS structure in the present investigation.

BHY is a slow-growing (doubling time of $\sim 72 \mathrm{~h}$ ) cell line, established from the tumour of the lower alveolus of a Japanese male, which was highly invasive towards the mandible and the oral floor (14). It has a stable growth on agarose, which means a reliable measure of the metastatic capability of the cancer cells (15). In the present study, BHY spheroids did not follow the typical growth patterns, with proliferating cells next to the oxygen supply and nutrients, and quiescent and necrotic cells farthest away from the capillaries, as described before (9).

However, a better understanding of the molecular mechanisms underlying tumour resistance to apoptotic cell death is expected to provide the basis for a rational approach to develop molecular targeted therapies (16).

Although BHY is a slow growing, difficult to cultivate highly differentiated OSCC cell line, it forms reproducible compact spheroids on an agarose surface. The generation of MCS using the liquid overlay technique is a simple method that can be used for further cancer research and anticancer drug development.

\section{References}

1. Friedrich J, Seidel C, Ebner R and Kunz-Schughart LA: Spheroid-based drug screen: Considerations and practical approach. Nat Protoc 4: 309-324, 2009.

2. Adcock AF, Trivedi G, Edmondson R, Spearman C and Yang L: Three-dimensional (3D) cell cultures in cell-based assays for in vitro evaluation of anticancer drugs. J Anal Bioanal Tech 6: 247, 2015.

3. Metzger W, Sossong D, Bächle A, Pütz N, Wennemuth G, Pohlemann T and Oberringer M: The liquid overlay technique is the key to formation of co-culture spheroids consisting of primary osteoblasts, fibroblasts and endothelial cells. Cytotherapy 13: 1000-1012, 2011.

4. Ivascu A and Kubbies M: Rapid generation of single-tumor spheroids for high-throughput cell function and toxicity analysis. J Biomol Screen 11: 922-932, 2006.

5. Santini MT and Rainaldi G: Three-dimensional spheroid model in tumor biology. Pathobiology 67: 148-157, 1999.

6. Mueller-Klieser W: Tumor biology and experimental therapeutics. Crit Rev Oncol Hematol 36: 123-139, 2000.

7. Timmins NE, Dietmair S and Nielsen LK: Hanging-drop multicellular spheroids as a model of tumour angiogenesis. Angiogenesis 7: 97-103, 2004.

8. Timmins NE and Nielsen LK: Generation of multicellular tumor spheroids by the hanging-drop method. Methods Mol Med 140: 141-151, 2007.

9. Yuhas JM, Li AP, Martinez AO and Ladman AJ: A simplified method for production and growth of multicellular tumor spheroids. Cancer Res 37: 3639-3643, 1977.

10. Kelm JM, Timmins NE, Brown CJ, Fussenegger $M$ and Nielsen LK: Method for generation of homogeneous multicellular tumor spheroids applicable to a wide variety of cell types. Biotechnol Bioeng 83: 173-180, 2003.

11. Lee C, Lee C, Atakilit A, Siu A and Ramos DM: Differential spheroid formation by oral cancer cells. Anticancer Res 34: 6945-6949, 2014.

12. Hagemann J, Jacobi C, Hahn M, Schmid V, Welz C, Schwenk-Zieger S, Stauber R, Baumeister P and Becker S: Spheroid-based 3D cell cultures enable personalized therapy testing and drug discovery in head and neck cancer. Anticancer Res 37: 2201-2210, 2017.

13. Gebhard C, Gabriel C and Walter I: Morphological and immunohistochemical characterization of canine osteosarcoma spheroid cell cultures. Anat Histol Embryol 45: 219-230, 2016.

14. Kawamata H, Nakashiro K, Uchida D, Harada K, Yoshida $H$ and Sato M: Possible contribution of active MMP2 to lymph-node metastasis and secreted cathepsin L to bone invasion of newly established human oral-squamous-cancer cell lines. Int J Cancer 70: 120-127, 1997.

15. Lin CJ, Grandis JR, Carey TE, Gollin SM, Whiteside TL, Koch WM, Ferris RL and Lai SY: Head and neck squamous cell carcinoma cell lines: Established models and rationale for selection. Head Neck 29: 163-188, 2007.

16. Fulda S: Tumor resistance to apoptosis. Int J Cancer 124: 511-515, 2009. 